\title{
Comparación de pruebas para medir la fatiga muscular en el entrenamiento de atletas hombres de CrossFit: una revisión sistemática Comparison of tests to measure muscle fatigue in training of male CrossFit athlete: a systematic review
}

\author{
Norma Hernández Nájera, Natanael Cervantes Hernández, Claudia Esther Carrasco Legleu \\ Universidad Autónoma de Chihuahua (México)
}

\begin{abstract}
Resumen. El CrossFit es un entrenamiento sumamente practicado por diversos atletas alrededor del mundo, los cuales se preparan cada año para ser mejores y ser seleccionados para competir en los CrossFit Games. Actualmente se conoce muy poco acerca de las pruebas que valoran la fatiga muscular en atletas que practique este tipo de entrenamiento. La fatiga muscular tiene un papel fundamental en el rendimiento de los atletas para su preparación y competición. El propósito de este estudio fue comparar las diferentes pruebas que se utilizan para medir la fatiga muscular en atletas hombre de CrossFit. Por ellos se realizó una búsqueda sistemática siguiendo las pautas de PRISMA y en los artículos se incluyeron los siguientes criterios de inclusión: artículos originales, atletas hombres de CrossFit y evaluación de la fatiga muscular. Estudios publicados en los últimos 10 años (desde el año 2011 hasta el 31 de marzo del 2021), los artículos no se limitaron a un idioma en específico, encontrando en su mayoría inglés y español. La selección principal fue por medio del título, después por medio del resumen y lectura del documento completo en estudios potenciales. Finalmente, se encontraron cinco artículos en los cuales se identificaron diversas pruebas para medir la fatiga muscular en los atletas de CrossFit. Como conclusión dada la amplia variedad de entrenamientos (WOD), tipos de ejercicios e intensidades en CrossFit es necesario valorar pruebas físicas y marcadores bioquímicos.
\end{abstract}

Palabras clave: CrossFit, fatiga muscular, atletas hombres, valoración, alto rendimiento.

\begin{abstract}
CrossFit is training highly practiced by various athletes around the world, who prepare each year to be better and be selected to compete in the CrossFit Games. Currently very little known about the tests that assess muscle fatigue in athletes who practice this type of training. Muscle fatigue plays a fundamental role in the performance of athletes for their preparation and competition. The purpose of this study was to compare the different tests used to measure muscle fatigue in male CrossFit athletes. For them, a systematic search was carried out following the PRISMA guidelines, and the following inclusion criteria were included in the articles: original articles, CrossFit male athletes, and evaluation of muscle fatigue. Studies published in the last 10 years (from 2011 to March 31, 2021) the articles were not limited to a specific language, finding mostly English and Spanish. The main selection was by title, then by abstracting and reading the entire document in potential studies. Finally, five articles were found in which various tests were found to measure muscle fatigue in CrossFit athletes. In conclusion, given the wide variety of workouts (WOD) types of exercises and intensities in CrossFit, it is necessary to assess physical tests and biochemical markers.
\end{abstract}

Keywords: CrossFit, fatigue muscle, athletes, assessment, high performance.

\section{Introducción}

El CrossFit es una modalidad de entrenamiento creada por Greg Glassman en 1995 en los Estados Unidos. Este deporte ha tenido un incremento exponencial en su práctica en todo el mundo con este incremento se consolida esta modalidad como un deporte muy peculiar. Pocos estudios se han centrado en los determinantes del éxito competitivo en CrossFit, tanto morfológicamente como en rendimiento (Barbieri et

Fecha recepción: 04-06-21. Fecha de aceptación: 09-09-21

Natanael Cervantes Hernández

ncervantes@uach.mx al., 2017). Este deporte exige gran demanda para el cuerpo humano, tanto a nivel físico, metabólico inclusive a nivel psicológico, por lo que la evaluación del rendimiento, por medio de pruebas físicas implica gran importancia para esta disciplina (Mangine et al., 2020).

El programa de CrossFit es utilizado para desarrollar fuerza y acondicionamiento físico el cual comprende 10 capacidades físicas: resistencia cardiovascular/respiratoria, factor de resistencia, stamina, fuerza, flexibilidad, potencia, velocidad, coordinación, agilidad, equilibrio y precisión (Gutierrez, 2010). Este entrenamiento consiste en realizar movimientos funcionales de forma variada y ejecutados a alta intensidad, los elementos que principalmente se utilizan son ejercicios de gimna- 
sia avanzada (lagartijas paradas de manos, caminar de manos, anillos), ejercicios de levantamiento de peso (peso muerto, sentadilla trasera, arranque, envión) y actividades cardiovasculares (correr, remar, saltar la cuerda). La metodología de CrossFit depende de la plena divulgación de métodos, resultados y críticas, el cual respalda sus valores por medio de una plataforma en internet y pagina web, es de acceso abierto, lo que hace que entrenadores y atletas se vuelvan co-desarrolladores a través de una comunidad en línea espontánea y cooperativa (CrossFit, 2020).

Un documento de consenso elaborado por el Consortium for Health and Military Performance (CHAMP) y el American Collage of the Sport Medicine (ACSMpor sus siglas en inglés) asoció una posible aparición de un alto riesgo de lesiones con programas como CrossFit (Bergeron et al., 2011). Esta situación de sobrecarga no solo trae consigo lesiones, sino que está asociada a la aparición de fatiga temprana, estrés oxidativo, menor resistencia a la contracción muscular provocado por la intensidad del ejercicio, la velocidad de ejecución y el tiempo de la sesión, así como una mayor percepción del esfuerzo y ejecución de movimientos inseguros, todo esto posterior al ejercicio repetitivo afectando al rendimiento físico. (Claudino et al., 2018). La fatiga muscular según Gosker (2008), se define como la disminución de la capacidad para generar cantidades adecuadas de fuerza o potencia muscular durante un periodo de ejercicio y/o entrenamiento. La fatiga muscular puede generarse por múltiples factores como: alteraciones del $\mathrm{pH}$, la temperatura y la acumulación de productos del metabolismo celular en el flujo sanguíneo (especialmente de los que resulta de la hidrólisis del adenosintrifosfato (ATP), como el adeonosíndifosfato ADP, adenosínmonofosfato (AMP), inosinamonofosfato (IMP), grupo de fosfato inorgánico (Pi) y amonio. La pérdida de esta homeóstasis del ión $\mathrm{Ca}^{+}$, y el papel de la cinética de algunos iones en los medios intra y extracelulares (como el $\mathrm{K}+, \mathrm{Na}^{+}$y Cl$\mathrm{Mg}^{+}$) (Gómez et al., 2010), generando una menor disponibilidad de sustratos energéticos en el músculo activo durante los periodos de entrenamiento (Ascensão et al., 2003).

Debido a lo anterior es de suma importancia que los entrenadores e investigadores desarrollen programas de entrenamiento con un diseño que incluya pruebas físicas especializadas en el deporte y marcadores bioquímicos para la evaluación de la fatiga muscular e identificar algún daño previo alguna competencia y/o para mejorar técnicas de recuperación y maximizar el entrenamiento y posteriormente el rendimiento (Finsterer, 2012). Para contribuir a la mejora de los programas de entrenamiento de CrossFit es importante tener seguimiento y evaluación, esto con el fin de tener una adaptación eficaz y un volumen de entrenamiento adecuado para lograr reducir el riesgo de lesiones y optimizar el rendimiento deportivo (Peguero et al., 2019).

Aunque hay una gran cantidad de participantes de CrossFit, la evidencia que existe en la sobrecarga de los atletas elite en cuanto a sus entrenamientos y/o competencias aun es escaza. Por lo tanto, el propósito de este estudio fue analizar los hallazgos encontrados en la literatura científica relacionada con CrossFit y fatiga muscular a través de una revisión sistemática.

\section{Metodología}

\section{Límites de búsqueda}

Para realizar la revisión sistemática se siguió la declaración PRISMA Preferred Reporting Items for Systematic reviews and Meta-Analyses (Urrútia \& Bonfill, 2010). Se llevó a cabo una búsqueda en las bases de datos: Pubmed, Web of Science y Scopus entre los años 2011 al 2021. La búsqueda finalizo el 31 de marzo del 2021. Se seleccionaron estas bases de datos porque son relevantes en el área de interés. Asimismo, los artículos que recogen estas bases de datos están publicados en revistas indexadas en el Journal Citation Report (JCR) o el Scimago Journal Rank (SJR). La estrategia de búsqueda utilizada en cada base de datos se muestra en la tabla 1.

\begin{tabular}{|c|c|}
\hline Base de datos & Estrategia de búsqueda \\
\hline Pubmed & $\begin{array}{l}\text { CrossFit AND fatigue muscle, Athletes IN CrossFit, CrossFit AND } \\
\text { assessment, high performance AND CrossFit }\end{array}$ \\
\hline Web of Sciencie & $\begin{array}{l}\text { CrossFit AND fatigue muscle, Athletes IN CrossFit, CrossFit AND } \\
\text { assessment, high performance AND CrossFit }\end{array}$ \\
\hline Scopus & $\begin{array}{l}\text { CrossFit AND fatigue muscle, Athletes IN CrossFit, CrossFit AND } \\
\text { assessment, high performance AND CrossFit }\end{array}$ \\
\hline
\end{tabular}

\section{Criterios de selección}

Se establecieron los siguientes criterios de inclusión: artículos originales, atletas hombres de CrossFit y evaluación de la fatiga muscular. Los artículos no se limitaron a un idioma en específico, encontrando en su mayoría inglés y español. La selección fue por medio del título, después por medio del resumen y lectura del documento completo. Cuando se aplicaron los criterios de inclusión se fueron seleccionando los artículos elegibles con el fin de refinar la búsqueda y encontrar los artículos potenciales, como lo muestra la figura 1. Después 
de dar lectura al título y/o resumen se identificaron 19 artículos elegibles de los cuales 14 fueron eliminados por duplicación, seleccionando 5 artículos potenciales.

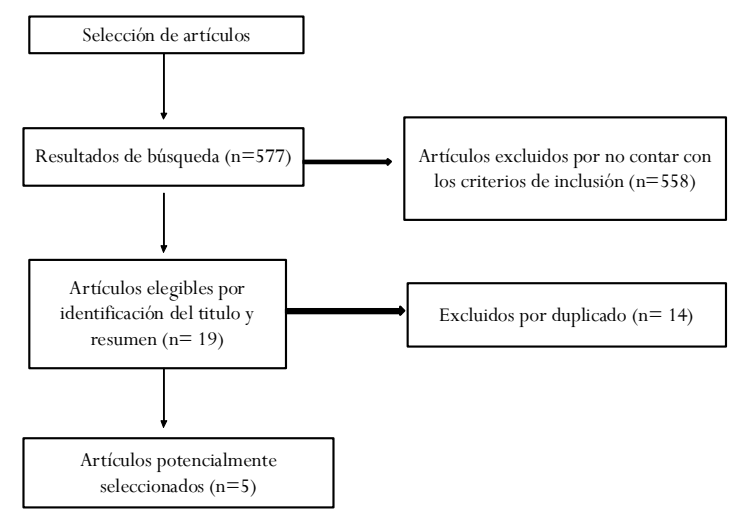

Figura 1. Diagrama de selección de artículos mediante los criterios PRISMA.

\section{Resultados}

Los artículos descartados fueron de temas diversos relacionados con el CrossFit, pero no incluían los criterios de inclusión que fueron: artículos originales, atletas hombres de CrossFit y evaluación de la fatiga muscular. Por otra parte, el título, objetivo y/o metodología no tenían relación con el objetivo de la revisión sistemática. El trabajo de Murawska et al., (2015) con el estudio relacionado con factores neuronales. En el estudio de Kellman (2010) con prevención del sobre entrenamiento. Sousa et al., (2016) con el estudio sobre diferencia entre individuos entrenados para competición e individuos que practican por salud. Pritchard et al., (2020) con prácticas del entrenamiento en atletas. García y
Toledo, (2019) con la medición de una valoración del perfil funcional. Bellar et al., (2015) con la relación entre capacidad aerobia y potencia máxima, Tibana et al., (2019) con la periodización del entrenamiento, otro estudio sobre prevalencia de lesiones durante el entrenamiento. Moya et al. (2017) en el área de nutrición y suplementación de (Jaramillo et al., 2017; Gogojewicz et al., 2020) y factores psicológicos como el estudio de Cain et al., (2020) entre otros.

Una vez hecho el filtrado se obtuvieron cinco artículos que cumplían con todos los criterios de inclusión. En la tabla 2 se muestran los datos descriptivos de los artículos incluidos. Se puede extraer con mayor detalle los resultados expuestos en cada uno de los trabajos, según el objeto de estudio, muestra, pruebas de evaluación para la fatiga muscular, hallazgos importantes y otras pruebas mencionadas.

\section{Objeto de estudio}

De los cinco artículos incluidos, Maté et al., (2017) y en el (2018) muestran una evaluación a los niveles de lactato en sangre y respuesta a la fatiga muscular inducidos por diferentes entrenamientos de CrossFit, mientras que Dexheimer et al., (2019) determinó que medida de rendimiento fisiológico es el mayor indicador en el entrenamiento de CrossFit. Otro de los artículos analizó el rendimiento físico y parámetros bioquímicos (nitrógeno ureico en sangre, bilirrubina total, transaminasa GOT, transaminasa GPT, lactato deshidrogenasa, creatina fosfoquinasa y glucosa), después de dos entrenamientos de CrossFit y su recuperación después de 48 horas (Timón et al., 2019). Por últi-

Tabla 2.

\begin{tabular}{|c|c|c|c|c|c|}
\hline Autor/año & Objetivo & Muestra & $\begin{array}{l}\text { Pruebas de evaluación para fatiga } \\
\text { muscular }\end{array}$ & Otras pruebas & Hallazgos importantes \\
\hline Maté et al. 2017 & $\begin{array}{l}\text { Evaluar los niveles de fatiga muscular } \\
\text { inducidos por las tres modalidades } \\
\text { de CrossFit WOD; gimnasia (G), } \\
\text { acondicionamiento metabólico (M) y } \\
\text { levantamiento de pesas (W). }\end{array}$ & $\begin{array}{l}\text { rn }=34 \text { hombres. } \\
\text { Edad: } 22.03 \pm \\
3.1 \text { años } \\
\text { y }\end{array}$ & $\begin{array}{l}\text { Evaluacion de lactato en sangre. } \\
\text { Fatiga muscular durante una } \\
\text { countermovement jump (CMJ) } \\
\text { realizada en una plataforma de } \\
\text { fuerza portatil }\end{array}$ & $\begin{array}{l}\text { Gymnastics WOD. Metabolic } \\
\text { conditioning WOD. Power clean } \\
\text { incremental load test. Weightlifting } \\
\text { WOD. }\end{array}$ & $\begin{array}{l}\text { El nivel de lactato en sangre despues de las sesiones de } \\
\text { gimnasia }(\mathrm{G}) \text { y levantamiento de peso (W) fue mayor que al } \\
\text { final de la sesion de condicion metabolico. La alta intensidad y } \\
\text { el volumen en } \mathrm{G} \text { y W wod podrían conducir a una rigidez } \\
\text { musculo-tendinosa que provoca pérdida de la capacidad de } \\
\text { salto }\end{array}$ \\
\hline $\begin{array}{l}\text { Dexheimer et al. } \\
2019\end{array}$ & 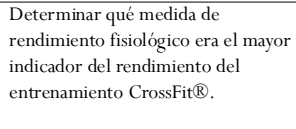 & $\begin{array}{l}\mathrm{n}=12 \text { hombres. } \\
\text { Edad: } 18-45 \\
\text { años. }\end{array}$ & Wingate Anaerobic Test (WAnT) & $\begin{array}{l}\text { Body Composition and } \\
\text { Anthropometrics. Graded Exercise } \\
\text { Test 3-Minute All-Out-Test Total Body } \\
\text { Strength_CrossFit }(\text { Total CrossFit } \mathbb{} \\
\text { Workouts }\end{array}$ & $\begin{array}{l}\text { Los atletas con una parte inferior del cuerpo más fuerte } \\
\text { pueden mantener un ritmo rápido con menos fatiga, } \\
\text { y completando el entrenamiento mas rápidamente. }\end{array}$ \\
\hline Timón et al. 201؟ & $\begin{array}{l}\text { 9Analizar los parámetros bioquímicos } \\
\text { y el rendimiento físico después de } \\
\text { dos modalidades de CrossFit WOD, } \\
\text { y evaluar la recuperación a las } 48 \\
\text { horas. }\end{array}$ & $\begin{array}{l}\mathrm{n}=12 \text { hombres. } \\
\text { Edad: } 30.4 \pm \\
5.37 \text { años }\end{array}$ & $\begin{array}{l}\text { Lactato en sangre Parametros } \\
\text { bioquimicos Fatiga muscular por } \\
\text { medio de Countermovement } \\
\text { jumps (CMJ) }\end{array}$ & $\begin{array}{l}\text { Anthropometric measurements 1RM } \\
\text { Power Clean test. Maximal } \\
\text { incremental test in cycle ergometer } \\
\text { Physical performance tests }\end{array}$ & $\begin{array}{l}\text { Las dos sesiones de CrossFit provocaron cambio significativos } \\
\text { en las transaminasas hépaticas, marcadores de daño muscular y } \\
\text { metabolico. Todos los niveles se recuperaron y volvieron a la } \\
\text { linea de base a las } 48 \text { horas. }\end{array}$ \\
\hline $\begin{array}{l}\text { Wiewelhove et al } \\
2015\end{array}$ & $\begin{array}{l}\text { 1.Investigar los cambios de diferentes } \\
\text { marcadores para la evaluación de la } \\
\text { fatiga y la recuperación en respuesta } \\
\text { al entrenamiento en intervalos de } \\
\text { alta intensidad (HIIT). }\end{array}$ & $\begin{array}{l}\mathrm{n}=11 \text { hombres } \\
\text { Edad: } 22.9 \pm 1.9 \\
\text { años }\end{array}$ & Parametros bioquimicos & $\begin{array}{l}\text { Incremental treadmill test. 30-15 } \\
\text { intermittent fitness test. Repeated } \\
\text { sprint ability test (RSA). Jump and } \\
\text { linear sprint tests. Muscle contractile } \\
\text { markers. Subjetive marker (escala } \\
\text { EVA). Training program (seis dia) }\end{array}$ & $\begin{array}{l}\text { Un programa HIIT de seis días indujo cambios significativos } \\
\text { en la prueba de capacidad de sprint (RSA)indica que el } \\
\text { programa de entrenamiento indujo un estado temporal de } \\
\text { fatiga. Se demostro que la capacidad de potencia y la fuerza } \\
\text { reactiva en la parte inferior del cuerpo, así como el tiempo de } \\
\text { contraccion del recto femoral y biceps son marcadores } \\
\text { potencialmente útiles. }\end{array}$ \\
\hline Maté et al. 2018 & $\begin{array}{l}\text { Respuestas de fatiga } \\
\text { cardiometabólica y muscular a } \\
\text { diferentes entrenamientos de } \\
\text { CrossFit. }\end{array}$ & $\begin{array}{l}\mathrm{n}=32 \text { hombres } \\
\mathrm{Edad}=21.75 \pm \\
2.54 \text { años }\end{array}$ & $\begin{array}{l}\text { Lactato en sangre Fatiga muscular } \\
\text { por medio de Countermovement } \\
\text { jumps (CMJ) }\end{array}$ & $\begin{array}{l}\text { Wod } 1 \text { Cindy (AMRAP } 20 \text { min: } 5 \\
\text { t pullups, } 10 \text { push up y } 15 \text { squats) Wod } \\
2 \text { skip rope double unders Wod } 3 \\
\text { Power cleans Escala de borg }(6-20)\end{array}$ & $\begin{array}{l}\text { La variable de lactato siempre excedio de } 10 \mathrm{mmol} / \mathrm{l} \text {, con una } \\
\text { diferencia significativa en el wod } 1 \text { con } 12.0 \pm 2.1 \mathrm{mmol} / \mathrm{l} \\
\text { esto se puede atribuir a los difetentes volúmenes de trabajo e } \\
\text { intervalos de descanso y que cualquier ejercicio de alta } \\
\text { intensidad, los períodos de recuperación son esenciales para } \\
\text { evitar la fatiga muscular }\end{array}$ \\
\hline
\end{tabular}


mo, Wiewelhove et al., (2015) investigaron los cambios con diferentes biomarcadores (creatina quinasa, proteína $\mathrm{C}$ reactiva y urea) para la evaluación de la fatiga y la recuperación en respuesta al entrenamiento de intervalos de alta intensidad (HIIT).

\section{Muestra}

Teniendo en cuenta el tamaño de la muestra las de mayor numero de sujetos son de 34 hombres con una edad de $22.03 \pm 3.1$ años Maté et al., (2017) y 32 hombres con una edad de $21.75 \pm 2.54$ años Maté et al., (2018) luego los estudio de Dexheimer et al., (2019) y Timón et al., (2019) coinciden en 12 hombres con una diferencia en las edades, Dexheimer et al., (2019) en un rango de edad entre 18 y 45 años, mientras que Timón et al., (2019) con $30.4 \pm 5.37$ años. Por último, Wiewelhove et al., (2015) es la muestra más pequeña con 11 hombres de 22.9 \pm 1.9 años.

\section{Pruebas de evaluación para fatiga muscular}

En lo que respecta a las pruebas utilizadas, tres de los estudios utilizan la prueba de Countermovement jumps (CMJ) Maté et al., (2017 y 2018) y Timón et al., (2019), de igual manera los mismos autores evaluaron lactato en sangre. Por otro lado, Dexheimer et al., (2019), utilizó la prueba de Wingate Anaerobic Test (Want) y por último en dos estudios Timón et al., (2019) y Wiewelhove et al., (2015) evaluaron parámetros bioquímicos.

\section{Otras pruebas}

Maté et al., (2017) utilizarón cuatro pruebas, gymnasticsWOD, metabolic conditioning WOD, power clean incremental load test y weightlifting WOD. Dexheimer et al., (2019) evaluaron body composition and anthropometrics, graded exercise test, 3-minuteallout-test, total body strength CrossFit $\mathbb{R}$ total y

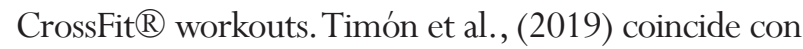
la evaluación de anthropometric measurements, evaluando otras tres pruebas mas, 1RM Power Clean test, maximal incremental test on a cycle ergometer y physical performance test. Por otro lado, Wiewelhove et al., (2015), utilizaron las siguientes pruebas incremental treadmill test, 30-15 intermittent fitness test, repeated sprint ability test (RSA), jump and linear sprint tests, muscle contractile markers, subjetive marker (escala EVA) y training program (seis dias). Por último, Maté et al (2018) utilizaron tres WOD del entrenamiento de CrossFit, WOD 1 Cyndy, WOD 2 skip rope, doublé under y WOD 3 power cleans, también se utilizó la escala de borg (6-12) para la percepción de esfuerzo.

\section{Hallazgos importantes}

Por último, teniendo en cuenta los hallazgos más importantes de cada estudio, se observó que en el estudio de Maté et al., (2017) la fatiga muscular se produjo después de las sesiones de gimnasia y levantamiento de peso y se observó que al final de la sesión de condición metabólica se recuperaba la fatiga en la evaluación de lactato. También se menciona que la alta intensidad y el volumen en las sesiones de gimnasia y levantamiento de peso podrían conducir a una rigidez musculo-tendinosa que provoca pérdida de capacidad de salto. Dexheimer et al., (2019) observaron que los atletas que tiene una parte inferior más fuerte pueden mantener un ritmo rápido con menos fatiga, completando los entrenamientos más rápidamente. En el estudio de Timón et al., (2019) las dos sesiones de CrossFit provocaron cambios significativos en los parámetros bioquímicos específicamente en las transaminasas hepáticas, marcadores de daño muscular y metabólico, sin embargo, todos los niveles se recuperaron y volvieron a la línea base a las 48 hrs. Por otra parte, Wiewelhove et al., (2015) encontraron que un programa de HIIT de seis días provoco cambios significativos en la prueba de capacidad de sprint (RSA) e indicando que el programa de entrenamiento indujo un estado temporal de fatiga. Se demostró que la capacidad de potencia y la fuerza reactiva en la parte inferior del cuerpo, así como el tiempo de contracción del recto femoral y bíceps son marcadores potencialmente útiles. Por último, el estudio de Maté et al., (2018) la variable de lactato siempre excedió de $10 \mathrm{mmol} / \mathrm{l}$, con una diferencia significativa en elWOD 1 con $12.0 \pm 2.1 \mathrm{mmol} / \mathrm{l}$ esto se puede atribuir a los diferentes volúmenes de trabajo e intervalos de descanso y que cualquier ejercicio de alta intensidad en los periodos de recuperación son esenciales para evitar la fatiga muscular.

\section{Discusión}

De los artículos que fueron descartados los temas con mayor relevancia en el CrossFit es referente a lesiones provocadas por el entrenamiento (Moya et al., 2017; Summitt et al., 2016; Minghelli, 2019; Gardiner et al., 2020; Weisenthal et al., 2014; Mehrab et al., 2017; Tafuri et al., 2019), los autores manifiestan la importancia de la incidencia de lesiones en el entrenamiento de CrossFit, así como identificar cuando fue la lesión y 
su causa. Por otro lado, el área de nutrición y suplementación tiene un papel importante para los atletas de CrossFit algunos autores (Jaramillo et al., 2017; Gogojewicz et al., 2020; Fogaça et al., 2020; Kramer et al., 2016; Stein et al., 2019) hacen énfasis en planes nutricionales y suplementación para mejorar el rendimiento de los atletas. En el área de psicología autores señalan algunas pruebas de evaluación para individuos que practican CrossFit, (Elks et al., 2020; Cadegiani et al., 2019; Cataldi et al., 2021). Por último, algunos estudios (Murawska et al., 2015; Kellman et al., 2010; Sousa et al., 2016; Pritchard et al., 2020; García y Toledo 2019; Bellar et al., 2015; Tibana et al., 2019) podrían tener un poco más de relación con el objetivo de la revisión sistemática los cuales podrían ser tomados en cuenta en una nueva revisión de la literatura para próximas investigaciones.

Para el propósito de nuestra investigación, los cinco artículos seleccionados utilizan diversas pruebas para medir la fatiga muscular en los atletas de CrossFit, lo cual indica que realizar evaluaciones periódicamente brinda un mejor control en su desempeño físico. Respecto a las pruebas utilizadas para medir la fatiga muscular, en los estudios de Maté et al., (2017 y 2018) y Timón et al., (2019) coinciden con la evaluación por medio de la plataforma de fuerza portátil counter movement jump (CMJ) herramienta fiable para medir la fatiga neuromuscular aguda como la fatiga a lo largo de alguna temporada de entrenamiento (Moreno, 2020). Estos encontraron resultados en el volumen de trabajo que se realiza en la parte inferior del cuerpo ya que su ejecución es por medio de un salto vertical (Bobbert et al., 1996). Sin embargo, es una herramienta para evaluar la potencia en tren inferior, en lo que respecta a los atletas de CrossFit, los cuales constantemente utilizan todo el cuerpo para desarrollar los entrenamientos la prueba de counter movemet jump (CMJ) solo mostraría una parte de su evaluación, quedando incompleta su valorización al no considerar la parte superior del cuerpo.

Maté et al., (2017) en su estudio valoraron lactato en sangre, lo cual en sus hallazgos principales se identificó que al termino de realizar el entrenamiento de las sesiones de gimnasia y levantamiento de peso se produjo un valor mayor de lactato en contraste con el entrenamiento de condición metabólico se observó que el lactato se recuperaba más rápido al final de la sesión. En el 2018, Maté et al., realizaron otro estudio donde la variable de lactato siempre excedió de $10 \mathrm{mmol} / \mathrm{l}$ en los tres entrenamientos que aplicaron a los atletas, y encontraron una diferencia significativa en el WOD de Cindy ( 5 pull-ups, 10 push-ups, 15 squats, AMRAP 20 min) con $12 \mathrm{mmol} / 1$ esto se puede atribuir a que no cuenta con ningún intervalo de descanso en su ejecución. Un elemento importante en el ejercicio de alta intensidad son los periodos de descanso los cuales son esenciales para evitar la fatiga (Vidal et al., 2016). Timón et al., (2019) en su estudio mostro niveles más bajos de lactato en sangre en el WOD 1 AMRAP (hacer el mayor número de repeticiones de Burpees y Toes to Bar incrementando repeticiones $(1-1,2-2,3-3 \ldots)$ en cinco minutos. Comparado con en el WOD 2 RFT (en el menor tiempo posible realizar tres rondas de 20 repeticiones de wall ball y 20 repeticiones de power clean con el $40 \%$ de 1RM) el cual tiene una gran diferencia en cuanto a tiempo de ejecución, intensidad y ejercicios a desarrollar, teniendo una mayor exigencia corporal.

Por otra parte, en la evaluación de parámetros bioquímicos los altos niveles iniciales de LDH y CPK (por encima de los valores normales de referencia) podrían indicar que los participantes en el estudio presentaban un cierto nivel de fatiga y daño muscular (Banfi et al., 2012), provocado por la práctica habitual de sesiones de CrossFit de alta intensidad. Cabe destacar que todos lo niveles se recuperaron y volvieron a su línea de base a las 48 hrs por lo tanto el estrés metabólico causado por ambas pruebas no fue tan alto como para inducir un estado patológico.

Por otro lado, Wiewelhove et al., (2015) en su estudio de HIIT señalan que diferentes parámetros bioquímicos están involucrados con diferentes cambios en los marcadores para la fatiga y con la respuesta a la recuperación del entrenamiento. En su estudio de seis días de entrenamiento se produjeron cambios significativos en la prueba de sprint (RSA) provocando un estado temporal de fatiga, además de las pruebas de rendimiento, se proponen mediciones de marcadores sanguíneo para controlar las condiciones de fatiga y recuperación. En el marcador de la creatina quinasa (CK) reaccionó al programa de HIIT mostrando una elevación promedio de > 1000 U L « 1 después del período de entrenamiento y una disminución a niveles casi iniciales luego del período de recuperación, (Zambrano et al. 2019). La actividad de la CK sérica refleja la tensión mecánica muscular del entrenamiento, ya que la CK se filtra al plasma desde las fibras del músculo esquelético cuando están dañadas, incluyendo el daño de la membrana y las alteraciones miofibrilares caracterizadas por la desorganización de los miofilamentos y la perdida de la integridad del disco Z (Nuzzo et al., 2008), provo- 
cando un daño parcial responsable de la disminución del rendimiento.

Otros estudios con atletas de deportes de equipo informaron un aumento de las concentraciones de CK después de periodos intensificados de entrenamiento o competencia, Meyer \& Meister (2011). Sin embargo, no se observaron cambios en la proteína $\mathrm{C}$ reactiva y la urea entre las mediciones pre y la pos-1 y pos-2. En este mismo estudio se valoró el dolor muscular de inicio retardado (DOMS) subjetivamente mediante escala (EVA) (Byrne et al., 2014). El DOMS aumento después del periodo de entrenamiento y disminuyo después de 72 horas de recuperación. Por los tanto las mediciones objetivas de CK y DOMS subjetiva son un conjunto de pruebas para identificar el potencial de daño muscular inducido por HIIT asociado con la fatiga y la recuperación observadas en los atletas de dicho estudio.

Según los resultados se concluye que los marcadores inflamatorios valorados no están directamente relacionados con el daño muscular. Sin embargo, investigar otros marcadores bioquímicos relacionados con el efecto del entrenamiento a alta intensidad contribuiría a complementar las evaluaciones en los atletas de CrossFit (Aguiar et al., 2021). Por último, en el estudio de Dexheimer et al., (2019) los cuales solo utilizaron la prueba anaeróbica más popular Wingate Anaerobic Test (WanT) la cual determina la potencia anaeróbica máxima y la capacidad para mantenerse (Jakovljevic et al. 2018), donde relaciona que los atletas con una parte inferior del cuerpo más fuerte pueden mantener un ritmo rápido con menos fatiga, completando el entrenamiento dosificado más rápidamente. No obstante, es interesante analizar y comparar las diferentes pruebas para medir fatiga muscular en diversos entrenamientos de CrossFit ya que nos muestran una gran variedad lo cual es parte de una planeación y dosificación del entrenamiento de manera adecuada y segura.

\section{Conclusiones}

Una vez realizada la revisión sistemática de la literatura, y teniendo en cuenta el objetivo de investigación marcado, es posible concluir, que los trabajos realizados en este campo del conocimiento utilizan diferentes pruebas y entrenamientos de CrossFit para medir y/o evaluar la fatiga muscular en los atletas hombres, lo cual podrían ayudar a los entrenadores y practicantes de CrossFit a controlar y planificar su entrenamiento adecuadamente para evitar situaciones de fatiga crónica y lesiones muscular.
Dada la amplia variedad de entrenamientos (WOD), tipos de ejercicio e intensidades en CrossFit es necesaria una mayor investigación para comprender con mayor profundidad las pruebas y/o valoraciones físicas como los marcadores bioquímicos involucrados en este tipo de entrenamiento.

\section{Referencias}

Aguiar, R. S., Lopes, G. C., de Castro, J. B. P., Prícipe, V. A., Mazini Filho, M. L., da Gama, D. R. N., \& Vale, R. G. (2021). Effects of high-intense resistance training on salivary cortisol in trained individuals: a systematic review (Efectos del entrenamiento de resistencia de alta intensidad sobre el cortisol salival en individuos entrenados: una revisión sistemática). Retos, (41), pp.265-271.

Ascensão, A., Magalhães, J., Oliveira, J., Duarte, J. A., \& Soares, J. (2003). Fisiologia da fadiga muscular. Delimitação conceptual, modelos de estudo e mecanismos de fadiga de origem central e periférica.

Banfi G., Colombini A., Lombardi G., Lubkowska A. (2012). Metabolic markers in sports medicine. Adv Clin Chem. 56: pp. 1-54.

Barbieri, J. F., Correia, R. F., Castaño, L. A. A., Brasil, D. V. C., \& Ribeiro, A. N. (2017). Comparative and correlational analysis of the performance from 2016 crossfit games high-level athletes. Manual Therapy, Posturology \& Rehabilitation Journal, 1-4.

Bellar, D., Hatchett, A., Judge, L. W., Breaux, M. E., \& Marcus, L. (2015). The relationship of aerobic capacity, anaerobic peak power and experience to performance in CrossFit exercise. Biology of sport, 32(4), p. 315.

Bergeron, M. F., Nindl, B. C., Deuster, P. A., Baumgartner, N., Kane, S. F., Kraemer, W. J., ... \& O'Connor, F. G. (2011). Consortium for Health and Military Performance and American College of Sports Medicine consensus paper on extreme conditioning programs in military personnel. Current sports medicine reports, 10(6), pp.383-389.

Bobbert, M. F., Gerritsen, K. G., Litjens, M. C., \& Van Soest, A. J. (1996). Why is countermovement jump height greater than squat jump height?. Medicine and science in sports and exercise, 28, pp.1402-1412.

Byrne C., Twist C., Eston R. (2004). Neuromuscular function after exercise-induced muscle damage: theoretical and applied implications. Sports Med. 34(1) pp.49-69.

Cadegiani FA, Kater CE, Gazola M. (2019). Clinical 
and biochemical characteristics of high-intensity functional training (HIFT) and overtraining syndrome: findings from the EROS study (The EROS-HIFT). J Sports Sci. Jun;37(11) pp. 1296-1307.

Caino, P. C. G., \& Martino, M. B. P. (2020). Predicción de los factores psicológicos de la ejecución deportiva según el Flow en practicantes de Crossfit. Revista de Psicología Aplicada al Deporte y al Ejercicio Físico, 5(2).

Cataldi S, Francavilla VC, Bonavolontà V, De Florio O, Carvutto R, De Candia M, Latino F, Fischetti F. (2021). Proposal for a Fitness Program in the School Setting during the COVID 19 Pandemic: Effects of an 8-Week CrossFit Program on PsychophysicalWellBeing in Healthy Adolescents. Int J Environ Res Public Health.Mar 18;18(6).

Claudino, J. G., Gabbett,T. J., Bourgeois, F., de Sá Souza, H., Miranda, R. C., Mezêncio, B., ... \& Serrão, J. C. (2018). CrossFit overview: systematic review and meta-analysis. Sports medicine-open, 4(1), pp.1-14.

CrossFit inc., (2020). Guía de entrenamiento del nivel 1. CrossFit training.

Dexheimer, J. D., Schroeder, E. T., Sawyer, B. J., Pettitt, R. W., Aguinaldo, A. L., \& Torrence, W. A. (2019). Physiological performance measures as indicators of crossfit ${ }^{\circledR}$ performance. Sports, 7(4), p.93.

Elks W, Jaramillo-Huff A, Barnes KL, Petersen TR, KomesuYM. (2020). The Stress Urinary Incontinence in CrossFit (SUCCeSS) Study. Female Pelvic Med Reconstr Surg.Feb;26(2):pp.101-106.

Finsterer, J. (2012). Biomarkers of peripheral muscle fatigue during exercise. BMC musculoskeletal disorders, 13(1), pp.1-13.

Fogaça LJ, Santos SL, Soares RC, Gentil P, Naves JP, Dos Santos WD, Pimentel GD, Bottaro M, Mota JF. (2020). Effect of caffeine supplementation on exercise performance, power, markers of muscle damage, and perceived exertion in trained CrossFit men: a randomized, double-blind, placebo-controlled crossover trial. J Sports Med Phys Fitness.Feb;60(2): pp.181-188.

García, R. H., \& Toledo, D. (2019). Valoración del perfil funcional en atletas de CrossFit. Estudio Piloto. Acciónmotriz, (23), pp.100-108.

Gardiner B, Devereux G, Beato M. (2020). Injury risk and injury incidence rates in CrossFit. J Sports Med Phys Fitness. Jul;60(7):pp. 1005-1013.

Gogojewicz, A., Eliwicka, E., \& Durkalec-Michalski, K. (2020). Assessment of dietary intake and nutritional status in crossfit-trained individuals: A descriptive study. International journal of environmental research and public health, 17(13), p.4772.

Gómez-Campos, R., Cossio-Bolaños, M. A., Brousett Minaya, M., Hochmuller-Fogaca, R.T., \& UNESCO, C. (2010). The mechanisms involved in acute fatigue. Revista Internacional de Medicina y Ciencias de la Actividad Física y del Deporte, 10(40), pp.537-555.

Gosker HR, Schols AM: Fatigued muscles in COPD but no finishing line in sight. Eur Respir J 2008, 31 : pp.693-694.

Gutiérrez, F. G. (2011). Conceptos y clasificación de las capacidades físicas. Cuerpo, CulturaY Movimiento, 1(1), pp.77-86.

Jakovljeviæ, D. K., Eric, M., Jovanovic, G., Dimitric, G., Cupic, M. B., \& Ponorac, N. (2018). Explosive muscle power assessment in elite athletes using wingate anaerobic test. Revista Brasileira de Medicina do Esporte, 24(2), pp.107-111.

Jaramillo, J. G., Loor, C. P., Aragundi, M. P., Méndez, P. L., Fernández, K. L., Escobar, E. B., \& Figueira, J.V. (2017). Sobre el riesgo de abuso de ayudas ergogénicas en los atletas de CrossFit. Revista Cubana de Alimentación y Nutrición, 27(2), p.15.

Kellmann, M. (2010). Preventing overtraining in athletes in high intensity sports and stress/recovery monitoring. Scandinavian journal of medicine \& science in sports, 20, pp.95-102.

Kramer SJ, Baur DA, Spicer MT, Vukovich MD, Ormsbee MJ. (2016). The effect of six days of dietary nitrate supplementation on performance in trained CrossFit athletes. J Int Soc Sports Nutr. Nov 3;13:39. Mangine, G. T., Tankersley, J. E., McDougle, J. M., Velazquez, N., Roberts, M. D., Esmat, T. A., ... \& Feito, Y. (2020). Predictors of CrossFit Open Performance. Sports, 8(7), pp. 102.

Martínez-Gómez, R., Valenzuela, P. L., Alejo, L. B., Gil-Cabrera, J., Montalvo-Pérez, A., Talavera, E., \& Barranco-Gil, D. (2020). Physiological Predictors of Competition Performance in CrossFit Athletes. International Journal of Environmental Research and Public Health, 17(10), pp. 3699.

Maté-Muñoz, J. L., Lougedo, J. H., Barba, M., CañueloMárquez, A. M., Guodemar-Pérez, J., GarcíaFernández, P., \& Garnacho-Castaño, M. V. (2018). Cardiometabolic and muscular fatigue responses to different crossfit ${ }^{\circledR}$ workouts. Journal of sports science \&medicine, 17(4), p.668.

Maté-Muñoz, J. L., Lougedo, J. H., Barba, M., GarcíaFernández, P., Garnacho-Castaño, M. V., \& Domínguez, R. (2017). Muscular fatigue in response to different modalities of CrossFit sessions. PloS 
one, $12(7)$.

McMullen, C. A., Hayeß, K., \& Andrade, F. H. (2005). Fatigue resistance of rat extraocular muscles does not depend on creatine kinase activity. BMC physiology, 5(1), pp.1-9.

Mehrab M, de Vos RJ, Kraan GA, Mathijssen NMC. (2017). Injury Incidence and Patterns Among Dutch CrossFit Athletes. Orthop J Sports Med.Dec $18 ; 5(12)$.

Meyer T, Meister S. (2011). Routine blood parameters in elite soccer players. Int J Sports Med. Nov;32(11) pp.875-81.

Minghelli B, Vicente P. (2019). Musculoskeletal injuries in Portuguese CrossFit practitioners. J Sports Med Phys Fitness. Jul;59(7) pp.1213-1220

Montesdeoca, A. J. Z., Párraga, J. Z. R., Chávez, M. B. T., \& Cedeño, N. V. (2019). Concentración sérica de creatina-quinasa y funcionalismo renal en adultos de centros de entrenamiento físico de Calceta. Dominio de las Ciencias, 5(1) pp.818-843.

Moreno, S. M. (2020). La altura del salto en contramovimiento como instrumento de control de la fatiga neuromuscular: revisión sistemática. Retos: nuevas tendencias en educación física, deporte y recreación, (37), pp.820-826.

Murawska-Cialowicz, E., Wojna, J., \& Zuwala-Jagiello, J. (2015). Crossfit training changes brain-derived neurotrophic factor and irisin levels at rest, after wingate and progressive tests, and improves aerobic capacity and body composition of young physically active men and women.J Physiol Pharmacol, 66(6), pp.811-821.

Nuzzo, J. L., McBride, J. M., Cormie, P., \& McCaulley, G. O. (2008). Relationship between countermovement jump performance and multijoint isometric and dynamic tests of strength. The Journal of Strength \& Conditioning Research, 22(3), pp.699-707.

Pérez, R. P., Despaigne, G. M. R., \& Manfugas, A. L. (2019). Metodología para la gestión de indicadores deportivos en centros de alto rendimiento de la Provincia de Santiago de Cuba. Revista Plus Economía, 7(1), pp.4-12.

Pritchard, H. J., Keogh, J.W., \& Winwood, P.W. (2020). Tapering practices of elite CrossFit athletes. International Journal of Sports Science \& Coaching, 15(5-6), pp.753-761.

Sousa, A. F., dos Santos, G. B., dos Reis, T., Valerino, A. J., Del Rosso, S., \& Boullosa, D. A. (2016). Differences in Physical Fitness between Recreational CrossFit $\AA$ and ResistanceTrained Individuals. Journal of Exercise Physiology Online, 19(5).

Stein JA, Ramirez M, Heinrich KM. The Effects of Acute Caffeine Supplementation on Performance in Trained CrossFit Athletes. Sports (Basel). 2019 Apr 25;7(4)p.95

Summitt RJ, Cotton RA, Kays AC, Slaven EJ (2016). Shoulder Injuries in Individuals Who Participate in CrossFit Training. Sports Health. Nov/Dec;8(6) pp. 541-546

Tafuri S, Salatino G, Napoletano PL, Monno A, Notarnicola A. (2019). The risk of injuries among CrossFit athletes: an Italian observational retrospective survey. J Sports Med Phys Fitness.Sep;59(9): pp.1544-1550.

Tibana, R. A., Sousa, N. M. F. D., Grigoletto, M. E. D. S., \& Voltarelli, F. A. (2019). Periodização do treinamento aplicada aos programas de condicionamento extremo. Rev. andal. med. deporte, pp. 300-306

Timón, R., Olcina, G., Camacho-Cardeñosa, M., Camacho-Cardenosa, A., Martinez-Guardado, I., \& Marcos-Serrano, M. (2019). 48-hour recovery of biochemical parameters and physical performance after two modalities of CrossFit workouts. Biology of sport, 36(3), pp. 283.

Urrútia, G., \& Bonfill, X. (2010). Declaración PRISMA: una propuesta para mejorar la publicación de revisiones sistemáticas y metaanálisis. Medicina clínica, 135(11), 507-511.

Vidal Inglés, D., Valero Valenzuela, A., \& SánchezAlcaraz Martínez, B. J. (2016). Efectos del Entrenamiento Interválico de Alta Intensidad en Corredores. Revisión Sistemática-Revista de Entrenamiento Deportivo. Revista de Entrenamiento Deportivo, 30(3).

Weisenthal BM, Beck CA, Maloney MD, DeHaven KE, Giordano BD. (2014). Injury Rate and Patterns Among CrossFit Athletes. Orthop J Sports Med. Apr 25;2(4).

Wiewelhove, T., Raeder, C., Meyer, T., Kellmann, M., Pfeiffer, M., \& Ferrauti, A. (2015). Markers for routine assessment of fatigue and recovery in male and female team sport athletes during high-intensity interval training. PloS one, 10 (10). 